\title{
3D-printed porous Ti6AI4V scaffolds for long bone repair in animal models: a systematic review
}

Yifei Gu ${ }^{1,2}$, Yi Sun ${ }^{1,2}$, Sohaib Shujaat ${ }^{1,2}$, Annabel Braem ${ }^{3}$, Constantinus Politis ${ }^{1,2}$ and Reinhilde Jacobs ${ }^{1,2,4^{*}}$ (D)

\begin{abstract}
Background: Titanium and its alloys have been widely employed for bone tissue repair and implant manufacturing. The rapid development of three-dimensional (3D) printing technology has allowed fabrication of porous titanium scaffolds with controllable microstructures, which is considered to be an effective method for promoting rapid bone formation and decreasing bone absorption. The purpose of this systematic review was to evaluate the osteogenic potential of 3D-printed porous Ti6AI4V (Ti64) scaffold for repairing long bone defects in animal models and to investigate the influential factors that might affect its osteogenic capacity.

Methods: Electronic literature search was conducted in the following databases: PubMed, Web of Science, and Embase up to September 2021. The SYRCLE's tool and the modified CAMARADES list were used to assess the risk of bias and methodological quality, respectively. Due to heterogeneity of the selected studies in relation to protocol and outcomes evaluated, a meta-analysis could not be performed.
\end{abstract}

Results: The initial search revealed 5858 studies. Only 46 animal studies were found to be eligible based on the inclusion criteria. Rabbit was the most commonly utilized animal model. A pore size of around 500-600 $\mu \mathrm{m}$ and porosity of 60-70\% were found to be the most ideal parameters for designing the Ti64 scaffold, where both dodecahedron and diamond pores optimally promoted osteogenesis. Histological analysis of the scaffold in a rabbit model revealed that the maximum bone area fraction reached $59.3 \pm 8.1 \%$ at weeks $8-10$. Based on micro-CT assessment, the maximum bone volume fraction was found to be $34.0 \pm 6.0 \%$ at weeks 12 .

Conclusions: Ti64 scaffold might act as a promising medium for providing sufficient mechanical support and a stable environment for new bone formation in long bone defects.

Trail registration The study protocol was registered in the PROSPERO database under the number CRD42020194100.

Keywords: Titanium alloy, Ti6Al4V, 3D printing, Animal study, Bone tissue engineering

\section{Background}

Since the early 1970 s, titanium and its alloys have been widely incorporated in the biomedical field for manufacturing implants and repairing bone defects. These alloys offer a lower Young's modulus compared to other

\footnotetext{
*Correspondence: Reinhilde.Jacobs@ki.se

${ }^{4}$ Department of Dental Medicine, Karolinska Institutet, Stockholm, Sweden

Full list of author information is available at the end of the article
}

materials, such as stainless steel, cobalt-chromium alloys and tantalum. Nevertheless, an elastic modulus mismatch still exists between titanium and bone tissue which could lead to bone atrophy, fracture, osteoporosis and early implant failure due to the stress shielding effect. To overcome this limitation, porous titanium scaffolds have been designed for reducing the modulus and mimicking the strength of natural bone, thereby allowing prevention of the stress shielding $[1,2]$ and promotion of scaffold 
fixation into the surrounding tissues $[3,4]$. The interconnected pores of the scaffold also offer a shorter healing time with an improved vascularization and exchange of nutrients. At present, porous titanium scaffolds have been employed in the manufacturing of three-dimensional (3D)-printed implants such as artificial lumbar fusion cages and acetabular joints [5-8]. These implants offer high compressive strength, bone-like elastic modulus, and promote long-term bone ingrowth. Furthermore, they have an optimal wear resistance for resisting scratches, cracks, and peeling [9]. These characteristics make porous titanium-based implants an excellent candidate for repairing bone defects.

Currently, porous titanium- 6 aluminium- 4 vanadium (Ti6Al4V) scaffold is one of the most commonly used materials for manufacturing load-bearing implants and repairing bone defects owing to its superior mechanical properties and osseointegration compared to commercially pure titanium and other alloys [10]. Many conventional material processing methods have been employed for fabricating porous Ti6Al4V scaffolds, which include sintering [11], solid-state foaming [12], and polymeric sponge replication [13]. In comparison, recent introduction of additive manufacturing (AM) technology and processes, such as selective laser melting (SLM) and electron beam melting (EBM), has allowed the fabrication of customizable 3D-printed porous Ti6Al4V (Ti64) scaffolds which offer predictable and predetermined unit cells. Both the aforementioned technologies belong to the powder bed fusion category, where the heat generated from an energy source (SLM: fiber laser, EBM: electronic beam) is used to selectively melt and fuse powder layer by layer based on the computer-aided design (CAD) model. When one layer of powder has been selectively melted, the build platform is lowered to a predetermined distance and the next layer is deposited. The process is repeated with each successive layer until the desired part is entirely constructed [14].

These 3D-printed porous scaffolds have the potential to replace other bone graft substitutes (allograft, autograft) for treating long bone defects, which are prone to certain risks such as restricted blood supply, disease transmission, and high morbidity rate. The key to reducing these risks is to utilize a porous Ti64 scaffold with interconnected pores having sufficient pore size $(>100 \mu \mathrm{m})$, which has the ability to promote cell proliferation and migration, as well as allow generation of new bone and capillaries [15]. Additionally, these scaffolds have a high coefficient of friction against cancellous bone $(\mu=1.09)$, which ensures a stable environment for new bone formation [16]. At an early stage following implantation, porous scaffolds also provide mechanical support to the damaged hard tissue [17].
Although the advantages of Ti64 scaffold have been well documented, it is biologically inert and lacks osteoinductivity. Consequently, it allows new bone formation only from the edges where it is in contact with the preexisting bone, which leads to a delayed complete fill-up of the defect. Ideally, the new bone should start forming at the center of the scaffold. Many studies have attempted to improve the osteogenic effect and fixation of Ti64 scaffold to the surrounding bone for increasing its long-term success rate, which include surface modification techniques (etching, nano-structuring, coating) and addition of growth factors [18]. However, clinical trials involving the implantation of a functional Ti64 porous scaffold are still rare, owing to the technique sensitivity and high costs.

Animal models are the most effective method for confirming the osteogenic potential of these modified functional Ti64 scaffolds by investigating the macroscopic and microscopic changes of the bone environment during osseointegration [19]. While testing the modified Ti64's osteogenic performance, it is common to use pristine Ti64 scaffold as a control group [4, 20-22], which allows exclusion of any inherent impact from other scaffold modifying materials. However, no data exist confirming the osteogenic properties of a pristine Ti64 scaffold, which in turn could impact the testing process with biased outcomes. It is also crucial to design the experiments more efficiently. For instance, if an experiment time period is set for too long, then both the control and experimental group might show equally distributed bone formation due to overgrowth, making it difficult to analyze the osteogenic differences between both groups without any discernible contrast. Furthermore, there is still room for improvement in AM technology for reducing excessive residual stress and surface roughness of the scaffold. Therefore, it is necessary to review the state of existing Ti64 scaffold's manufacturing and preclinical testing to better understand its osteogenic potential and yield more effective strategies for improving its clinical applicability.

The following systematic review aimed to report the current evidence related to the application of Ti64 scaffold for repairing long bone defects in animal models and to investigate the potential influential factors that might affect its osteogenic ability. The scope of this systematic review is shown in Fig. 1.

\section{Methods}

The study protocol was registered in the PROSPERO database under the number CRD42020194100. The systematic review followed the Preferred Reporting Items for Systematic Reviews and Meta-Analyses (PRISMA) guidelines. Two researchers (GYF, SY) searched the 


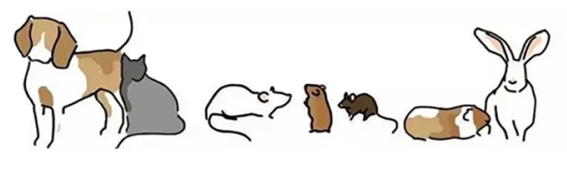

Animal models

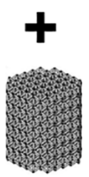

Ti64 scaffold II

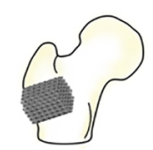

Osteogenesis in long bone defect

Fig. 1 Scope of the systematic review
Review questions

\section{Most relevant animal model?}

\section{Most optimal designing parameters?}

\section{Osteogenic result at different experimental periods?}

electronic databases of PubMed, Embase, and Web of Science for relevant studies published till September, 2021. Search keywords were ("Bone/bone regeneration/ bone reconstruction") AND ("Titanium alloy") AND ("3D printing"). The detailed search strings are presented in Additional file 1. Grey literature and references within the selected studies were also screened. Identified studies were imported into Endnote online software (Thomson Reuters, Philadelphia, PA, USA) for removing duplicates.

Table 1 describes the inclusion and exclusion criteria. All animal studies which reported on the application of Ti64 scaffold for long bone defect repair were included. "Long bone" was defined as a bone consisting of a tubular shaft (diaphysis) and two extremities (epiphyses), and "Ti64 scaffold" referred to a 3D-printed structure with a network of fully interconnected pores [23].
Two researchers (GYF, SY) independently screened the relevant articles based on the titles and abstracts and then read the full text of the included studies. Any disagreement was resolved through consensus. If an agreement could not be reached, a third researcher (RJ) was consulted. Risk of bias was assessed according to the SYRCLE's tool [24], and the CAMARADES list (www. camarades.info) was used for determining the methodological quality of the included articles.

The extracted data included scaffold characteristics (scaffold size and shape, fabrication method, pore size, porosity), study characteristics (animal model, implantation time, bone defect), and the reported osteogenic outcomes (bone area, bone volume).

The PICO (Population, Intervention, Comparison, and Outcome) criteria were as follows [25]:

Table 1 Inclusion and exclusion criteria used in this study

\begin{tabular}{|c|c|}
\hline Inclusion criteria & Exclusion criteria \\
\hline \multirow{6}{*}{$\begin{array}{l}\text { All in vivo studies which reported on the application of Ti64 scaffold for } \\
\text { long bone defect repair }\end{array}$} & 1. Non-English papers \\
\hline & 2. Descriptive studies, in vitro studies, and clinical trials \\
\hline & 3. Studies that used materials other than Ti6Al4V \\
\hline & $\begin{array}{l}\text { 4. Partially porous Ti64 implant or Ti64 implant with only a textured surface } \\
\text { layer }\end{array}$ \\
\hline & $\begin{array}{l}\text { 5. Animal models with comorbidities (hypertension, diabetes, osteoporosis, } \\
\text { etc.) }\end{array}$ \\
\hline & 6. Not a long bone defect (defect in cranial bone, jaw bone, etc.) \\
\hline
\end{tabular}


Population: animals with long bone defect.

Intervention/exposure: application of Ti64 scaffold for repairing long bone defect.

Comparison: not applicable.

Outcome: quantitative assessment of the new bone tissue formation.

\section{Results}

Figure 2 illustrates the screening flowchart based on PRISMA guidelines, and the PRISMA checklist can be found in Additional file 2. The search strategy retrieved
5858 articles. Following removal of duplicates, title and abstract screening, and full-text reading, 46 studies were eligible to be included in the review ranging from year 2013 till 2021, with the majority articles being published in 2020 (Fig. 3).

\section{Quality of the included studies Risk of bias}

Figure 4 illustrates the findings of the SYRCLE's risk of bias analysis. The allocation sequence of animals was adequately generated in 41 studies (89\%), and the baseline characteristics of the experimental animals were

\section{Identification of studies via databases and registers}

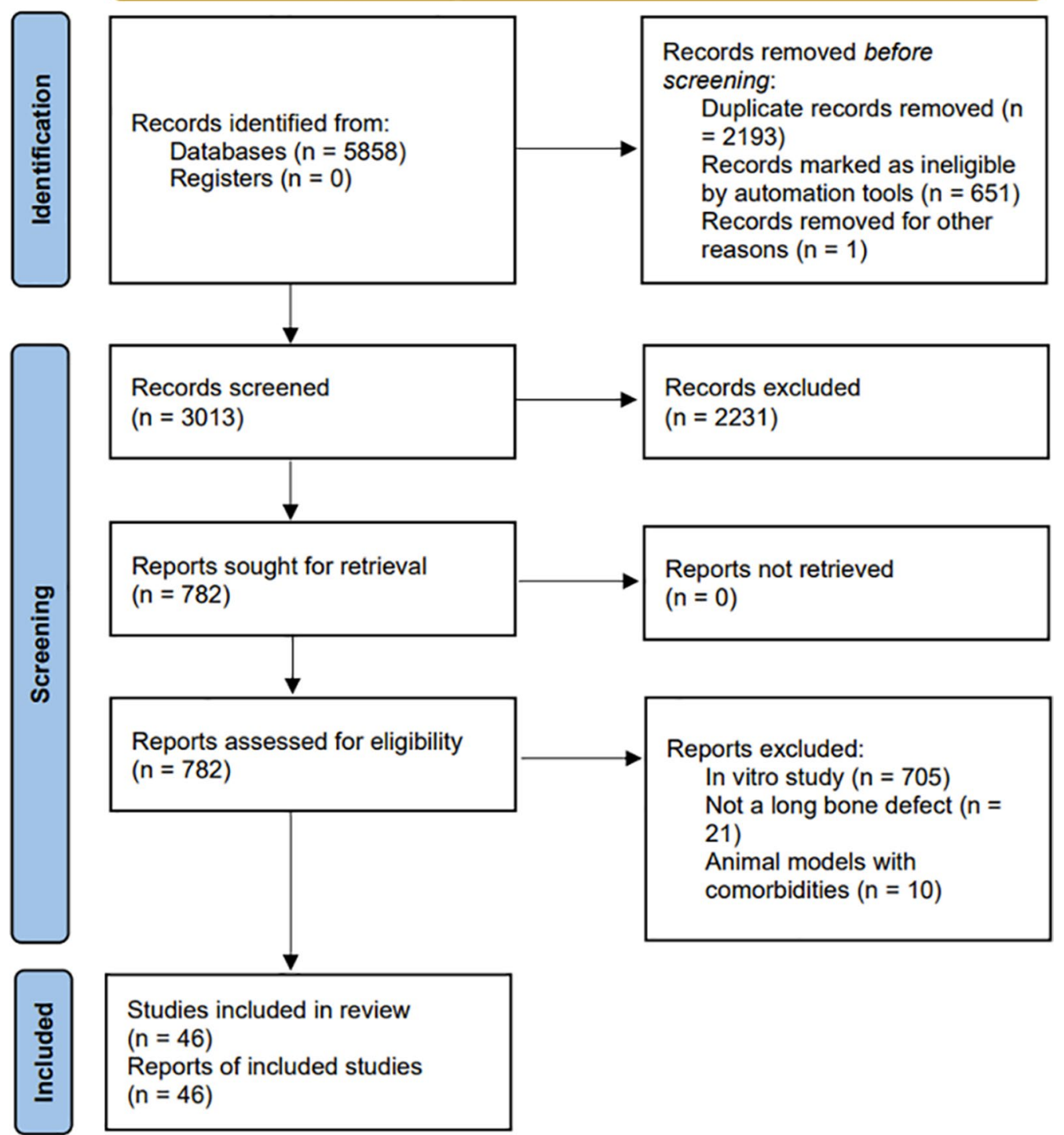

Fig. 2 Screening flow diagram based on the PRISMA guidelines 


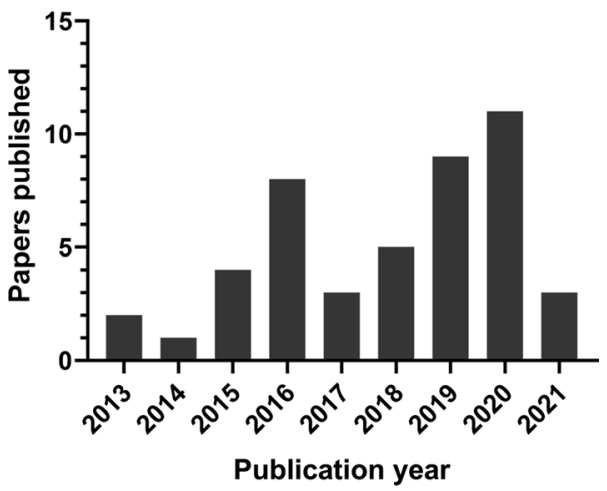

Fig. 3 Relationship between the year of publication and number of included articles

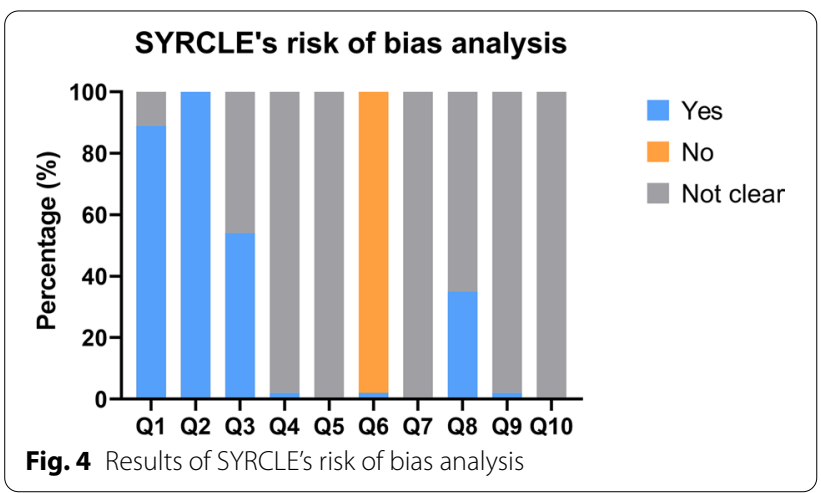

similar in all studies. No study mentioned whether the allocation was adequately concealed. Only one study stated that the animals were housed randomly. There was no explicit description of blind intervention or outcome evaluation. Most studies (45 articles, 98\%) did not select animals randomly for outcome assessment due to the high cost of animals. Additionally, many other items in the questionnaire were rated as "unclear," implying that reporting of these animal studies (mainly experimental designs) should be improved.

\section{Methodological quality}

Figure 5 illustrates the CAMARADES checklist-assisted methodological quality assessment. The outcomes of animal allocation, allocation concealment, and blind operation assessment were similar to those mentioned in "risk of bias" assessment. Animals in all the studies were healthy, and a neuroprotective anesthetic was administered in some studies (12 articles, $26 \%$ ). Only one article mentioned the sample size calculation method. Most studies (34 articles, 74\%) clarified the adherence to relevant operating guidelines during animal experiments. Furthermore, the rest of the items were rated as

\section{CAMARADES list of methodological} quality assessment

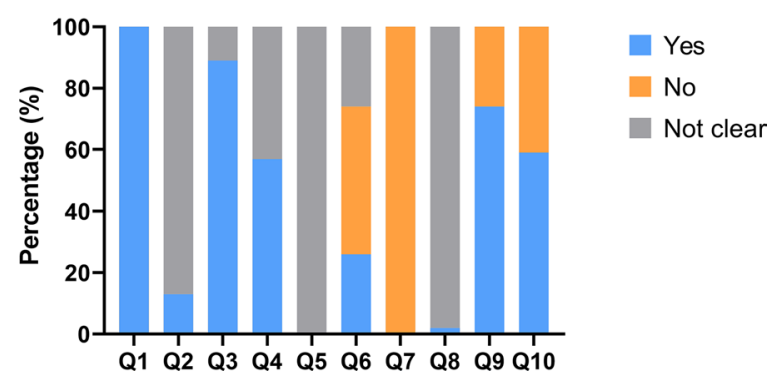

Fig. 5 Results of CAMARADES list of methodological quality assessment

"unclear" which matched the description as in "risk of bias" assessment.

\section{Characteristics of the included studies Scaffold design and animal study setup}

Table 2 summarizes the design of the included articles, and Fig. 6 illustrates a schematic workflow of a typical animal experiment. If an author's last name was the same in different articles, a number was used following the last name to distinguish the authors.

A total of 29 studies (63\%) mentioned the type of Ti6Al4V powder for 3D printing, with particle sizes ranging from 15 to $100 \mu \mathrm{m}$. In 16 studies, Grade 23 Ti6Al4V powder was incorporated. The $3 \mathrm{D}$ printing technique was mentioned in 44 studies (96\%), which included; SLM, (26 studies, 59\%), EBM (16 studies, 36\%), and selective laser sintering (SLS) (2 studies, 5\%). Post-processing of the Ti64 scaffold was mentioned in 30 studies (65\%), which involved removal of excess powder with either ultrasonic cleaning, sandblasting or acid treatment.

The design of choice for the scaffold was cylindrical in most studies (40 studies, $87 \%$ ) with the size variation depending on the animal model and reconstruction method. The strut size varied between 60 and $3600 \mu \mathrm{m}$, mostly ranging between 200 and $400 \mu \mathrm{m}$ (20 studies, $77 \%$ ). The reported pore size was $100-1500 \mu \mathrm{m}$, with the size of $500-700 \mu \mathrm{m}$ in the majority of studies (23 studies, 56\%), followed by $300-499 \mu \mathrm{m}$ (16 studies, 39\%). The porosity of Ti64 scaffold ranged from 25 to $90 \%$ in 40 studies; however, $60-70 \%$ was applied in most of the studies (22 studies, 55\%). Various pore shapes could be observed depending on the unit cell for designing the lattice structure, where some studies reported on more than one pore shape. Rhombic dodecahedron (8 studies, 27\%) and diamond (8 studies, $27 \%$ ) were the most commonly applied unit cell shapes. Other shapes included octahedron, tetrahedron, cube, 


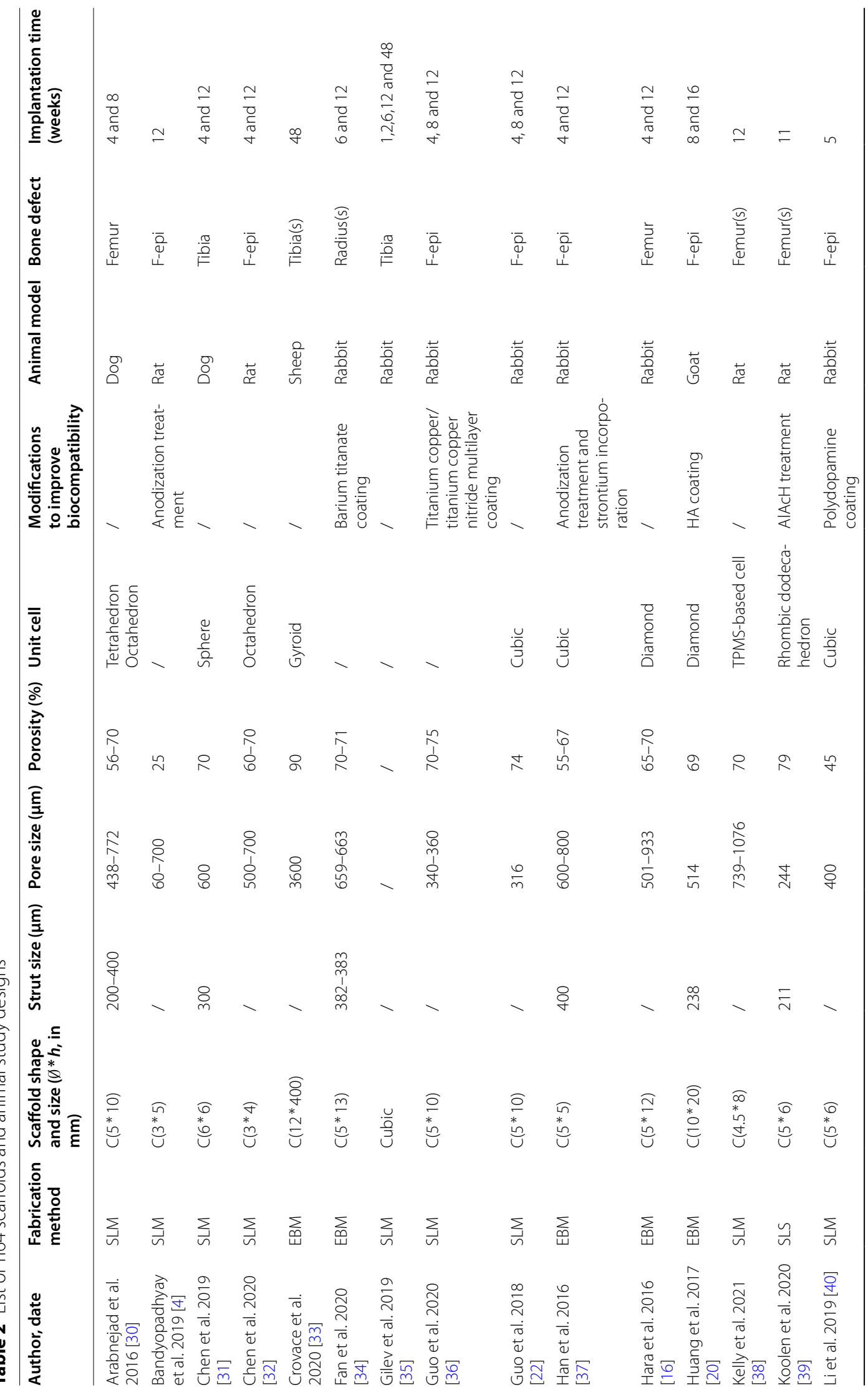




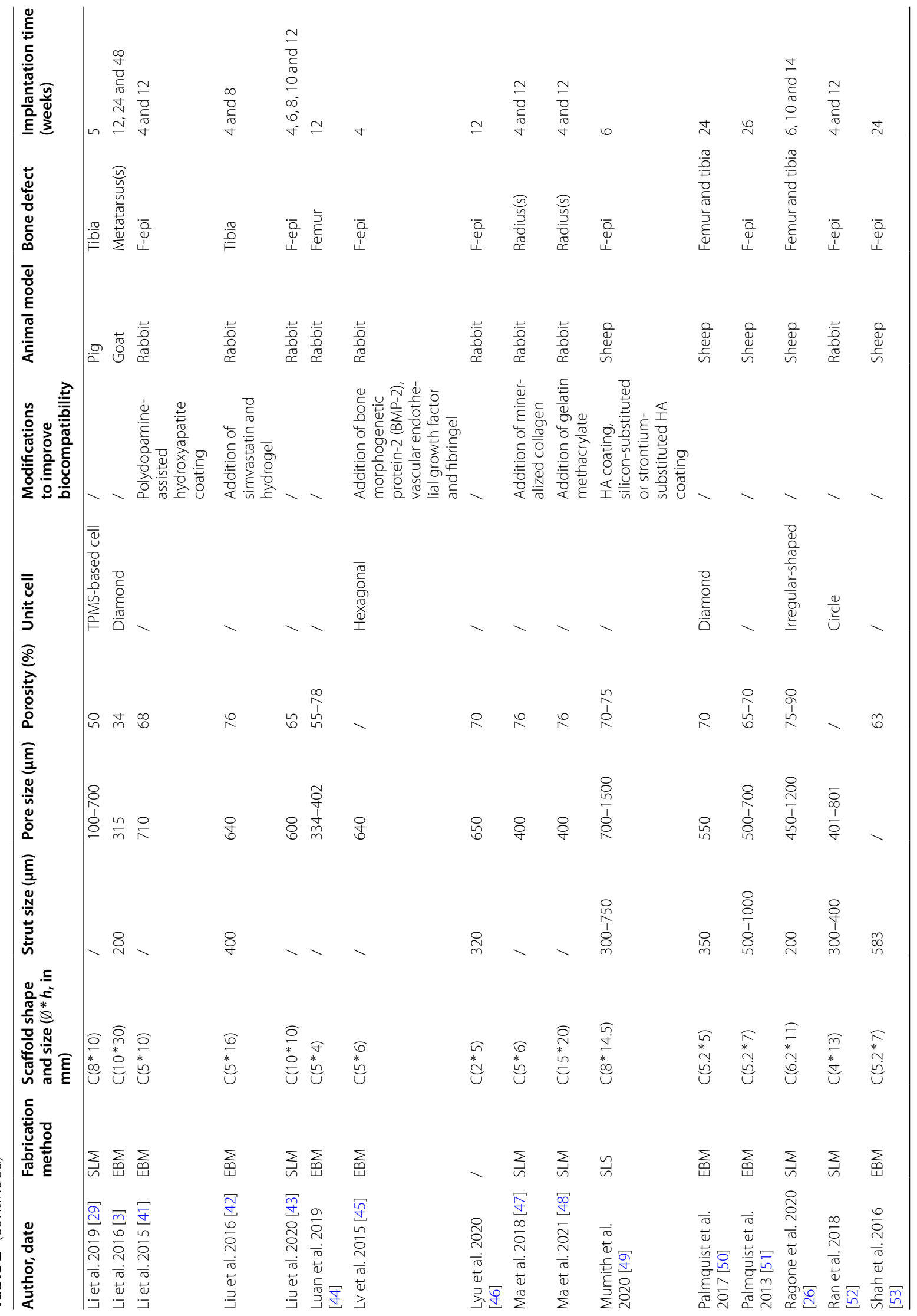




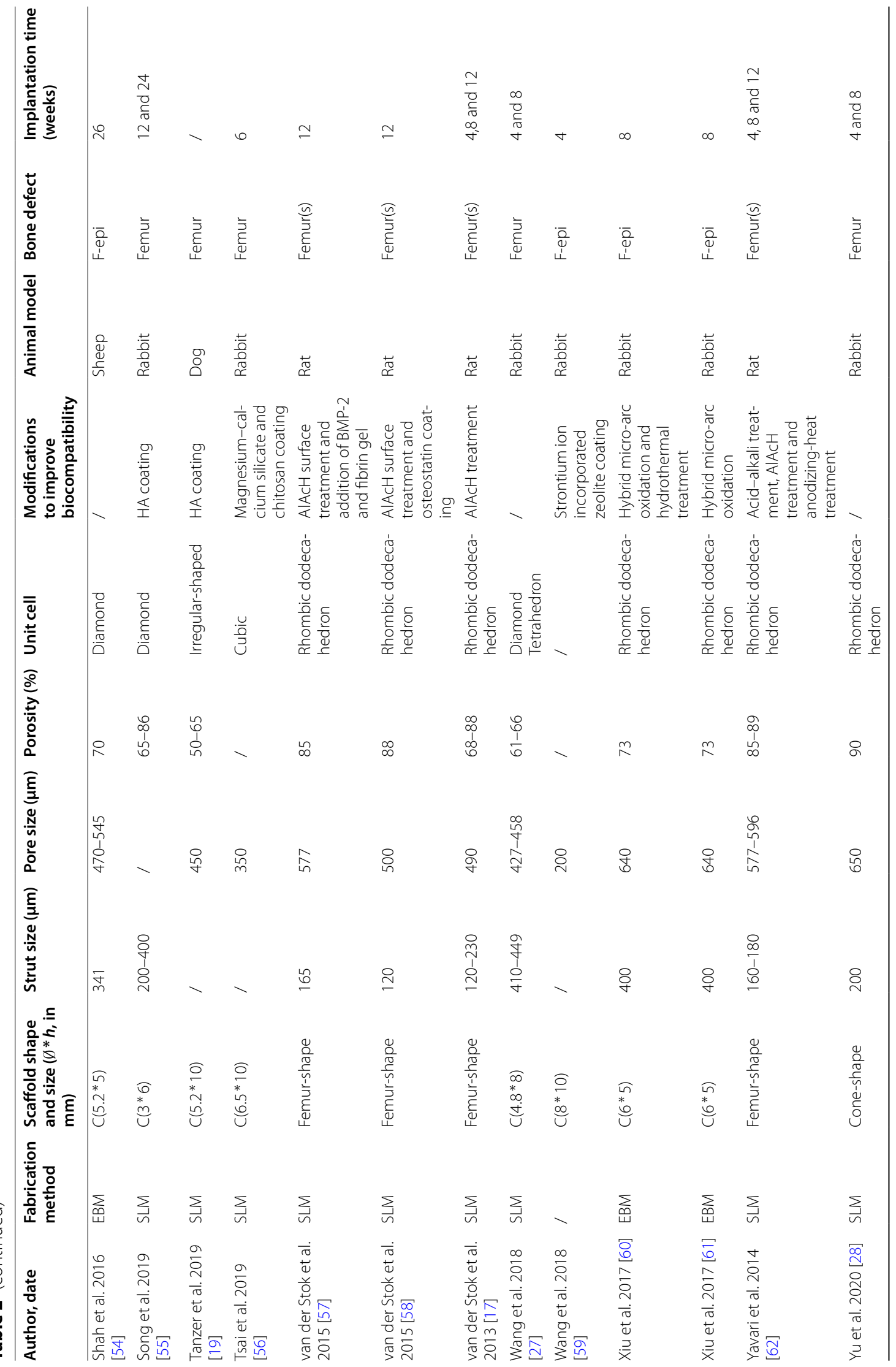




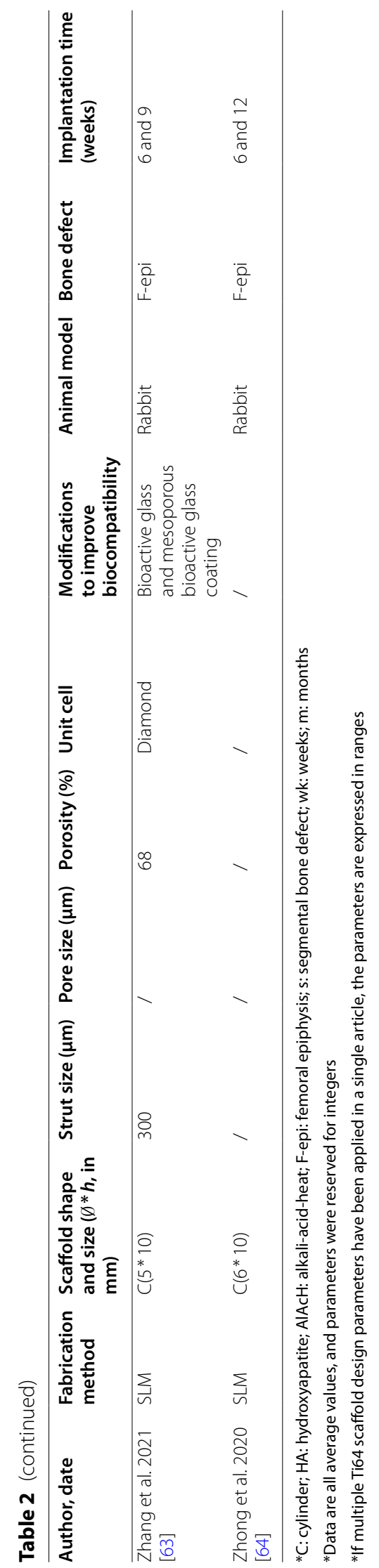




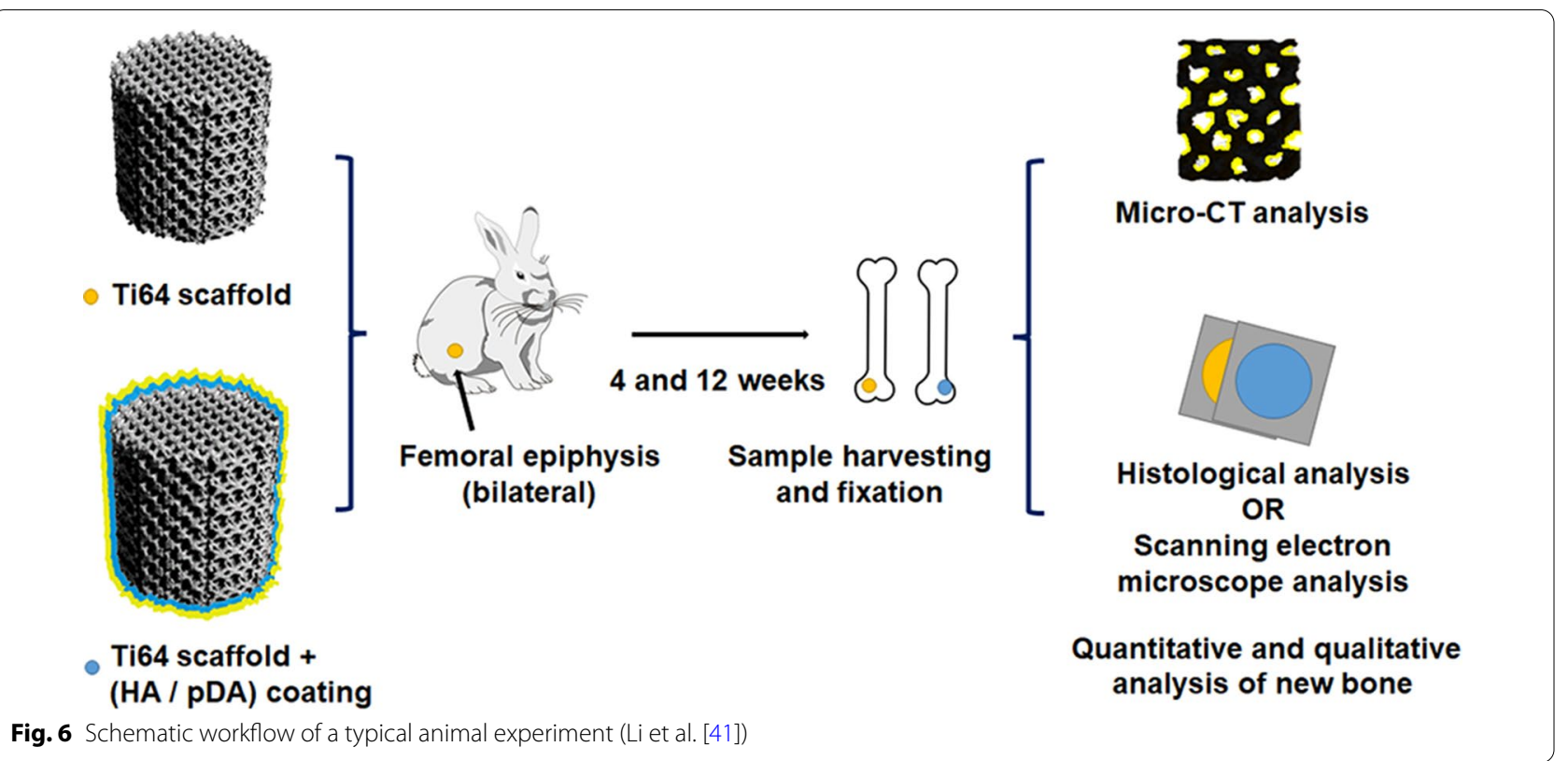

spiral tetrahedron, hexagon, and triply periodic minimal surface (TPMS)-based cells. Most studies (42 studies, $91 \%$ ) adopted a regularly arranged lattice structure, while others used a randomly generated or arranged irregular pore structure [19, 26-28].

In this review, the randomly distributed pore structures were divided into the following categories: 1 . Pore structure designed based on the Voronoi tessellation method [26]; 2. Pore structure designed using TPMS model [29]; 3. Randomly generated pore structure with varying pore sizes and shapes [19]; 4. Regularshaped and regular-sized pore structure with randomly arranged pores [27, 28].

The mechanical strength of the Ti64 scaffold was reported in 15 studies, ranging from 14 to $606 \mathrm{MPa}$, where 10 studies (66\%) described its strength between 30 and $200 \mathrm{MPa}$. The elastic modulus varied from 0.32 to $7.56 \mathrm{GPa}$, with the majority falling between 0 and 3 $\mathrm{GPa}$ (12 studies, $63 \%$ ).

Rabbit was the most commonly utilized animal model (25 articles, 54\%). Other animal models included rat, sheep, dog, goat and pig. Femur was the preferred implantation site in the majority of studies (36 studies, $78 \%$, femoral diaphysis: $n=15$, femoral epiphyses: $n=21$ ), while other sites included tibia and radius. Non-segmental bone defects accounted for most cases (35 studies, 76\%). The experiment duration ranged from 4 weeks to 12 months, and the follow-up time period for most articles was less than 12 weeks (36 studies, 78\%). Additionally, half of the studies assessed outcomes at two or more time-points (26 papers, 57\%).

\section{Bone tissue outcomes}

Quantitative evaluation of the new bone tissue formation was performed by either histomorphometric analysis, scanning electron microscopy (SEM) or 3D micro-CT assessment. The most commonly assessed two-dimensional (2D) and 3D parameters involved bone area fraction (BA/TA, new bone area over scaffold pore area) and bone volume fraction (BV/TV, new bone volume over scaffold pore volume), respectively. An overview of the outcomes is summarized in Tables 3 and 4. The addition of coating material, surface treatment, or other additives might alter the osteogenic performance of a Ti64 scaffold, hence, these conditions were not considered. Furthermore, segmental bone defects were excluded from the BA/TA and BV/TV analyses, as in such cases new bone only grows inward from both scaffold ends which results in a relatively low bone ingrowth compared to a single bone defect where most of the scaffold surface is covered by bone. Due to the heterogeneity in relation to study protocols and reported outcomes among the included studies, no meta-analysis could be performed.

\section{BA/TA analysis (Table 3 )}

The majority of BA/TA data were obtained from rabbit models (12/19 articles, 63\%), where scaffold was implanted either in the region of femoral diaphysis (5 articles) or femoral epiphysis (7 articles). The BA/TA in rabbit models ranged from $1.5 \pm 0.1 \%$ to $46.3 \pm 13.7 \%$ at weeks $4-6,8.2 \pm 2.3 \%$ to $59.3 \pm 8.1 \%$ at weeks $8-10,2.4 \pm 0.4 \%$ to $51.6 \pm 6.4 \%$ at weeks $12-14$, and $35.6 \pm 5.3 \%$ at weeks $24-26$ (only reported by Song et al. [55]). The maximum BA/TA at 
Table 3 Summary of reported BA/TA for pristine Ti64 scaffolds in the reviewed studies

\begin{tabular}{|c|c|c|c|c|c|c|}
\hline \multirow[t]{2}{*}{ Author, date } & \multirow{2}{*}{$\begin{array}{l}\text { Animal and bone } \\
\text { defect }\end{array}$} & \multirow[t]{2}{*}{ Group } & \multicolumn{4}{|l|}{ BA/TA (\%) } \\
\hline & & & 4-6 weeks & 8-10 weeks & 12-14 weeks & 24-26 weeks \\
\hline \multirow{2}{*}{$\begin{array}{l}\text { Arabnejad et al. } \\
2016 \text { [30] }\end{array}$} & \multirow[t]{2}{*}{ Beagle dog, femur } & Tetrahedron cell & $28.6 \pm 11.6$ & $41.3 \pm 4.3$ & / & / \\
\hline & & Octet truss cell & $35.5 \pm 1.9$ & $56.9 \pm 4$ & & \\
\hline Chen et al. 2019 [31] & Beagle dog, tibia & / & $11.9 \pm 2.2$ & / & $15.9 \pm 4.9$ & / \\
\hline Guo et al. 2020 [36] & Rabbit, f-epi & / & $11.6 \pm 1.9$ & $12.2 \pm 2.0$ & $24.1 \pm 3.0$ & / \\
\hline Guo et al. 2018 [22] & Rabbit, f-epi & / & $13.2 \pm 2.7$ & $35.6 \pm 2.7$ & $55.9 \pm 2.0$ & / \\
\hline \multirow[t]{2}{*}{ Han et al. 2016 [37] } & \multirow[t]{2}{*}{ Rabbit, f-epi } & Pore size $600 \mu \mathrm{m}$ & $2.3 \pm 0.4$ & / & $3.5 \pm 0.5$ & / \\
\hline & & Pore size $800 \mu \mathrm{m}$ & $1.5 \pm 0.1$ & / & $2.4 \pm 0.4$ & / \\
\hline \multirow[t]{4}{*}{ Hara et al. 2016 [16] } & \multirow[t]{4}{*}{ Rabbit, femur } & Pore size $500 \mu \mathrm{m}$ & $34.9 \pm 6.8$ & / & $50.1 \pm 8.3$ & / \\
\hline & & Pore size $640 \mu \mathrm{m}$ & $37.0 \pm 5.0$ & / & $50.9 \pm 6.7$ & / \\
\hline & & Pore size $800 \mu \mathrm{m}$ & $27.2 \pm 7.2$ & / & $51.6 \pm 6.4$ & / \\
\hline & & Pore size $1000 \mu \mathrm{m}$ & $34.7 \pm 8.4$ & / & $35.1 \pm 2.7$ & / \\
\hline Li et al. 2015 [41] & Rabbit, f-epi & / & $5.8 \pm 2.2$ & / & $12.2 \pm 2.2$ & / \\
\hline Lv et al. 2015 [45] & Rabbit, f-epi & / & $7.8 \pm 2.8$ & / & / & / \\
\hline \multirow[t]{2}{*}{$\begin{array}{l}\text { Palmquist et al. } \\
2017 \text { [50] }\end{array}$} & \multirow[t]{2}{*}{$\begin{array}{l}\text { Sheep, femur and } \\
\text { tibia }\end{array}$} & Scaffold in femur & / & / & / & $\begin{array}{l}\text { Central: } 26.5 \pm 9.2 \\
\text { Peripheral: } \\
57.2 \pm 10.9\end{array}$ \\
\hline & & Scaffold in tibia & / & / & / & $\begin{array}{l}\text { Central: } 45.6 \pm 19.5 \\
\text { Peripheral: } 8.0 \pm 10.4\end{array}$ \\
\hline $\begin{array}{l}\text { Palmquist et al. } \\
2017 \text { [51] }\end{array}$ & Sheep, f-epi & / & / & / & / & $44.7 \pm 4.4$ \\
\hline $\begin{array}{l}\text { Ragone et al. } 2020 \\
\text { [26] }\end{array}$ & $\begin{array}{l}\text { Sheep, femur and } \\
\text { tibia }\end{array}$ & / & $\begin{array}{l}\text { Cortical: } 75.0 \pm 13.5 \\
\text { Cancellous: } \\
27.0 \pm 15.0\end{array}$ & $\begin{array}{l}\text { Cortical: } 82.0 \pm 5.0 \\
\text { Cancellous: } \\
36.0 \pm 10.5\end{array}$ & $\begin{array}{l}\text { Cortical: } 82.0 \pm 9.0 \\
\text { Cancellous: } \\
51.0 \pm 14.0\end{array}$ & / \\
\hline Shah et al. 2016 [54] & Sheep, f-epi & / & / & / & / & $\begin{array}{l}\text { Central: } 32.9 \pm 4.8 \\
\text { Peripheral: } 60.0 \pm 4.6\end{array}$ \\
\hline Song et al. 2019 [55] & Rabbit, femur & / & / & / & $6.8 \pm 2.9$ & $35.6 \pm 5.3$ \\
\hline $\begin{array}{l}\text { Tanzer et al. } 2019 \\
\text { [19] }\end{array}$ & Beagle dog, femur & / & $41.5 \pm 8.2$ & / & $64.4 \pm 2.8$ & / \\
\hline Tsai et al. 2019 [56] & Rabbit, femur & / & $2.5 \pm 0.8$ & / & / & / \\
\hline \multirow{4}{*}{$\begin{array}{l}\text { Wang et al. } 2018 \\
\text { [27] }\end{array}$} & \multirow[t]{4}{*}{ Rabbit, femur } & Diamond cell (r) & $34.0 \pm 5.9$ & $36.3 \pm 1.0$ & / & / \\
\hline & & Diamond cell (ir) & $33.7 \pm 5.0$ & $36.8 \pm 2.3$ & / & / \\
\hline & & Diamond cell (g) & $30.2 \pm 3.3$ & $32.3 \pm 4.9$ & / & / \\
\hline & & Tetrahedron cell & $20.5 \pm 3.0$ & $24.3 \pm 1.9$ & / & / \\
\hline Xiu et al. 2017 [60] & Rabbit, f-epi & / & / & $8.2 \pm 2.3$ & / & / \\
\hline Xiu et al. 2017 [61] & Rabbit, f-epi & / & / & $10.8 \pm 3.4$ & / & / \\
\hline Yu et al. 2020 [28] & Rabbit, femur & / & $46.3 \pm 13.7$ & $59.3 \pm 8.1$ & / & / \\
\hline
\end{tabular}

*F-epi: femoral epiphysis; r: regularly distributed pores; ir: irregularly distributed pores; g: gradient distributed pores

*The data are all represented as means \pm standard deviations, and reserved for one decimal point

weeks 4 and 8 was only reported by Yu et al. [28]. Additionally, Hara et al. [16] observed that the depth of new bone ingrowth at week 12 exceeded $1.5 \mathrm{~mm}$ in a rabbit model.

Four studies provided BA/TA data in sheep models, and only Palmquist et al. [51] found that BA/TA reached $44.7 \pm 4.4 \%$ in femoral epiphysis at week 26 . The remaining three studies calculated the BA/TA at different scaffold regions (central part vs. peripheral part; cortical bone vs. cancellous bone). The new bone formation was significantly higher in the cortical bone and at the peripheral region of the Ti64 scaffold compared to cancellous bone and central area [26, 50,54].

Three studies assessed BA/TA at the region of femoral or tibial diaphysis in beagle dogs. It was found to be within the range of $11.9 \pm 2.2 \%$ to $41.5 \pm 8.2 \%$ at weeks $4-6,41.3 \pm 4.3 \%$ to $56.9 \pm 4.0 \%$ at weeks $8-10$ (only reported by Arabnejad et al. [30]), and $64.4 \pm 2.8 \%$ at weeks 12-14 (only reported by Tanzer et al. 2019 [19]). 
Table 4 Summary of reported BV/TV for pristine Ti64 scaffolds in the reviewed studies

\begin{tabular}{|c|c|c|c|c|c|c|}
\hline \multirow[t]{2}{*}{ Author, date } & \multirow[t]{2}{*}{ Animal and bone defect } & \multirow[t]{2}{*}{ Group } & \multicolumn{4}{|l|}{ BV/TV (\%) } \\
\hline & & & 4-6 weeks & 8-10 weeks & 12 weeks & 16 weeks \\
\hline \multirow[t]{6}{*}{ Chen et al. 2020 [32] } & \multirow[t]{6}{*}{ Rat, f-epi } & Porosity $60 \%$, pore size $500 \mu \mathrm{m}$ & / & / & $23.4 \pm 1.6$ & / \\
\hline & & Porosity $60 \%$, pore size $600 \mu \mathrm{m}$ & / & / & $21.0 \pm 2.1$ & / \\
\hline & & Porosity $60 \%$, pore size $700 \mu \mathrm{m}$ & / & / & $12.8 \pm 2.1$ & / \\
\hline & & Porosity $70 \%$, pore size $500 \mu \mathrm{m}$ & / & / & $23.2 \pm 1.8$ & / \\
\hline & & Porosity $70 \%$, pore size $600 \mu \mathrm{m}$ & / & / & $22.3 \pm 1.0$ & / \\
\hline & & Porosity $70 \%$, pore size $700 \mu \mathrm{m}$ & / & / & $18.3 \pm 1.4$ & / \\
\hline Guo et al. 2020 [36] & Rabbit, f-epi & / & $11.6 \pm 1.8$ & $17.1 \pm 1.6$ & $25.5 \pm 2.6$ & / \\
\hline \multirow[t]{2}{*}{ Han et al. 2016 [37] } & \multirow[t]{2}{*}{ Rabbit, f-epi } & Pore size $600 \mu \mathrm{m}$ & $8.4 \pm 1.3$ & / & $16.2 \pm 3.6$ & / \\
\hline & & Pore size $800 \mu \mathrm{m}$ & $4.3 \pm 1.0$ & l & $8.6 \pm 2.7$ & / \\
\hline Huang et al. 2017 [20] & Goat, f-epi & / & / & $5.1 \pm 1.8$ & / & $6.3 \pm 2.2$ \\
\hline Li et al. 2019 [40] & Rabbit, f-epi & / & 13.7 & / & / & / \\
\hline \multirow[t]{3}{*}{ Li et al. 2019 [29] } & \multirow[t]{3}{*}{ Pig, tibia } & Pore size $300-500 \mu m$ & $12.7 \pm 3.6$ & / & / & / \\
\hline & & Pore size $200-600 \mu m$ & $12.0 \pm 3.6$ & / & / & / \\
\hline & & Pore size $100-700 \mu m$ & $12.8 \pm 3.9$ & / & / & / \\
\hline Li et al. 2015 [41] & Rabbit, f-epi & / & $5.9 \pm 2.2$ & / & $11.0 \pm 2.6$ & / \\
\hline Liu et al. 2016 [42] & Rabbit, tibia & / & $26.7 \pm 1.0$ & $28.9 \pm 1.4$ & / & / \\
\hline \multirow[t]{3}{*}{ Luan et al. 2019 [44] } & \multirow[t]{3}{*}{ Rabbit, femur } & Porosity $55 \%$, pore size $334 \mu \mathrm{m}$ & / & / & $21.4 \pm 2.2$ & / \\
\hline & & Porosity $65 \%$, pore size $383 \mu \mathrm{m}$ & / & / & $24.6 \pm 2.0$ & / \\
\hline & & Porosity $78 \%$, pore size $400 \mu \mathrm{m}$ & / & / & $26.7 \pm 0.9$ & / \\
\hline Lyu et al. 2020 [46] & Rabbit, f-epi & / & / & / & $34.0 \pm 6.0$ & / \\
\hline Wang et al. 2018 [59] & Rabbit, f-epi & / & $6.0 \pm 0.2$ & / & / & / \\
\hline Yu et al. 2020 [28] & Rabbit, femur & / & $27.3 \pm 8.4$ & $29.8 \pm 2.2$ & / & / \\
\hline Zhang et al. 2021 [63] & Rabbit, f-epi & / & $13.4 \pm 1.0$ & $16.6 \pm 2.18$ & / & / \\
\hline Zhong et al. 2020 [64] & Rabbit, f-epi & / & $13.9 \pm 1.5$ & / & $16.0 \pm 1.3$ & / \\
\hline
\end{tabular}

*F-epi: femoral epiphysis

*The data are all represented as means \pm standard deviations, and reserved for one decimal point

\section{BV/TV analysis (Table 4)}

As BA/TA is limited to 2D sections, BV/TV based on micro-CT analysis provides a more comprehensive quantification of bone ingrowth. Rabbit model was applied in the majority of studies (11/14 articles, $79 \%)$ and the implantation site included femoral epiphysis (8 articles) and femoral diaphysis ( 3 articles. The BV/TV in rabbits ranged from $4.3 \pm 1.0 \%$ to $27.3 \pm 8.4 \%$ at weeks $4-6,16.6 \pm 2.18 \%$ to $29.8 \pm 2.2 \%$ at weeks $8-10$, and $8.6 \pm 2.7 \%$ to $34.0 \pm 6.0 \%$ at week 12. The maximum BV/TV at weeks $4-6$ and weeks 8-10 was only reported by Yu et al. [28]. Furthermore, the maximum BV/TV reached $12.8 \pm 3.9$ at weeks $4-6$ in pigs, $23.4 \pm 1.6 \%$ at week 12 in rats, and $6.3 \pm 2.2 \%$ at week 16 in goats.

\section{Variables affecting the osteogenic capacity of Ti64 scaffold}

From the selected studies and the data presented in Tables 3 and 4 , some variables have been summarized below, which might impact the osteogenic ability of a Ti64 scaffold:
Implantation site: Ragone et al. [26] found that the new bone formation in the cortical bone region was significantly greater compared to cancellous bone and the osseointegration almost completed after 2 months.

Implantation time: The maximum BA/TA values in rabbit models peaked at weeks $8-10$. The maximum BV/TV values in rabbit models and the maximum $\mathrm{BA} / \mathrm{TA}$ values in beagle dog models increased from week 4 till week 12 .

Pore size and porosity: Hara et al. [16] proposed that a pore size of $500-800 \mu \mathrm{m}$ was optimal for new bone growth. Similarly, Ran et al. [52] observed that a pore size of $600-800 \mu \mathrm{m}$ had greater osteogenic ability compared to a size of $400 \mu \mathrm{m}$. Furthermore, Chen et al. and Han et al. found that a scaffold with pore size of $500-600 \mu \mathrm{m}$ has more osteogenic capability compared to $700-800 \mu \mathrm{m}$ $[32,37]$. In terms of porosity, Luan et al. suggested that $78 \%$ porosity had a higher osteogenic capacity than $65 \%$ and $55 \%$ porosity. 
Pore shape: Within a Ti64 scaffold with high porosity (>50\%), Arabnejad et al. [30] observed that new bone formation in an octagonal shaped pore was more prominent compared to tetrahedral structure. Furthermore, Wang et al. [27] observed a lower bone formation with a tetrahedral pore structure compared to diamond shape.

\section{Discussion}

Over the past few years, animal experimentations for confirming the osteogenic potential of Ti64 scaffolds have gradually gained attention in the biomedical field. Therefore, the following review was conducted to accumulate evidence and report on the osteogenic ability of Ti64 scaffold for repairing long bone defects and to investigate influential factors which might impact its effectiveness. Meta-analysis could not be performed due to the presence of significant heterogeneity among the selected studies related to the scaffold design and size, defect type, and observation time. However, the qualitative evidence synthesis suggested certain critical commonalities and limitations associated within the the methodologies of the included studies for fabricating Ti64 scaffold with optimal osteogenic potential, which could act as a reference guide for future comparative studies.

When considering the animal model for the validation of scaffold, it is essential to understand the bone healing capacity of different animal species. Bone remodeling in small rodents is much faster than larger species [65]. Additionally, rabbits and dogs have a higher bone remodeling rate compared to humans. Therefore, it might be difficult to extrapolate the osteogenic response in these animals for a possible similar response in humans [66]. However, sheep, goat, and pig offer a similar bone remodeling rate to that of humans, making them a better choice for generating osteogenic responses and translating those findings to humans $[67,68]$.

In terms of the bone healing process, rats are considered less suitable based on the lack of haversian system. However, their bone remodeling is similar to the haversian remodeling in large animals [69]. Thereby, the absence of the haversian system should not be the sole reason for excluding rodents from studies where bone healing assessment is required. Furthermore, the bone healing process of dog, rabbit, sheep, and pig models is remarkably similar to that of humans [70,71].

The studies using a rat model in this review created segmental bone defects and the scaffold size was very small. We believe that rats should not be considered for assessing the performance of Ti64 scaffolds due to their size limitation and inability to insert multiple implants. Based on the International Organization for Standardization (ISO10993-6:2016(E)), the recommended implant size for biological evaluation in rabbits should be $2 \times 6 \mathrm{~mm}$ and $4 \times 12 \mathrm{~mm}$ in larger animals,such as sheep, goat, and $\mathrm{dog}$. The guidelines also recommend an observation time of 1 to 4 weeks' for assessing short-term outcomes and 12 weeks or more for long-term assessment. Additionally, the long-time follow-up time points for the animal models except rats should be $13,26,52,78$, and 104 weeks. However, the findings of the current review revealed that the experimental design in the majority of studies did not follow the international standards and had a follow-up period of less than 12 weeks, hence confirming a lack of evidence related to the standardized long-term outcome evaluation of Ti64 scaffold.

Rabbit was the most commonly applied animal model, and only a few studies assessed the osteogenic capacity of the scaffold with a large animal model such as sheep, goat, dog, and pig. In contrast with large models, small animals are easier to handle, less expensive, and appropriate for screening implant materials before testing in larger models. Unlike small animals, the large animal models have similar bone healing capabilities to that of humans. At the same instance, it should be kept in mind that every large animal model also has its pros and cons. For instance, dogs offer an optimal model for assessing Ti64 scaffold's effectiveness; however, due to ethical concerns and increased public scrutiny their application in animal research has been declining [72]. An adult sheep has similarities in weight, metabolism, and bone remodeling rates to that of humans and could be considered ideal for testing the scaffold and transferring the findings to a clinical setting [73]. However, researchers tend to use young animals due to financial constraints, which might underestimate the effectiveness of the scaffold as their bones have not fully matured.

In this review, most included studies adopted the femoral condyle defect model or the transcortical defect model. The femoral condyle defect model is most relevant to plastic surgery applications [74]. When there are many groups of scaffolds to be tested on the same animal model at the same time (for example, in the paper of Arabnejad et al. [30], there are four groups of scaffolds to be tested on the same animal model at the same time), the use of transcortical defects is a viable option for achieving greater consistency within the animal. Segmental bone defects were excluded from the review as they are more prone to failure due to bending or breaking of the fixation plate, screw failure, infection, and muscular and neurovascular damage. Additionally, inclusion of segmental defects would have led to bias within the findings of the review, as their mechanical integrity differs from that of a single defect which could negatively impact the osteogenic efficiency and functional recovery of the bone defect. 
Based on the findings of the review, Ti64 scaffold induced remarkable osteogenesis in the cortical region compared to cancellous bone. A possible explanation could be the penetration of a lower mechanical stimulus into the cancellous bone surrounding the scaffold [49]. Additionally, the cancellous bone has a randomly distributed pore structure, which cannot be replicated by the regularly distributed simple pattern. In contrast, the randomly distributed pore structures demonstrated higher permeability allowing optimal bone ingrowth $[19,28]$. The newly formed bone continues to grow over time with the maximum BA/TA and $\mathrm{BV} / \mathrm{TV}$ peaking at both weeks $4-6$ and weeks $8-10$ due to the osteogenic effect of the randomly distributed pores which outperform a simple topological distribution [19].

The osseointegration of Ti64 scaffold is primarily determined by the pore size, structure, porosity, and interconnectivity [19]. The pore size in the included studies ranged from 100 to $900 \mu \mathrm{m}$ [75]. A large pore size promotes the growth of blood vessels but reduces the mechanical properties and cell adhesion. On the contrary, a small pore size improves cell adhesion and tissue growth; however, the likelihood of pore blockage increases [76]. The findings of the review suggested that the pore sizes ranging from 500 to $600 \mu \mathrm{m}$ were found to be most optimal for an in vivo fabrication of Ti64 scaffold. Furthermore, an ideal porosity for porous scaffolds should be around $80-90 \%$ [76]. The majority of Ti64 scaffolds included in the review had a porosity of $60-70 \%$ for ensuring optimal mechanical strength. Furthermore, their compressive strength (33-193 MPa) and elastic modulus $(0.02-3 \mathrm{GPa})$ were equal to that of human cortical bone for avoiding the stress shielding effect [75]. A higher porosity than the aforementioned limit should be avoided to inhibit weakening of the scaffold's mechanical properties.

The pore shape of the Ti64 scaffold varied among studies without any consensus on which shape offered the most optimal outcomes. The most commonly applied shapes were diamond and rhombic dodecahedron. The diamond lattice is isotropic in nature with evenly distributed deformation, which helps to reduce stress concentration. Its structure is closely similar to that of cancellous bone and has a large curvature radius which induces higher tissue amplification [27]. Based on these benefits, a diamond lattice is widely incorporated in porous scaffolds [77]. On the other hand, rhombic dodecahedron lattice has a higher yield stress and is stable under multidirectional compression forces, thereby making it an ideal choice for manufacturing load-bearing implants [28, 78]. However, care should be taken that the dodecahedron shape is designed with only obtuse angles, as acutely angled pores are easily damaged during the melting stage of printing. Other designed pore shapes in the review involved tetrahedral, octahedral, gyroid, and TPMS lattices which are beneficial for improving the scaffold's strength and rigidity, isotropy, load resistance and surface area, and permeability, respectively [30, 32, 33, 38].

In this review, all Ti64 scaffolds were implanted immediately following the creation of bone defect, which is clinically applicable for procedures where sufficient preoperative time is available for treatment planning such as lumbar intervertebral fusion and acetabular joint reconstruction. An immediate insertion allows for a reduction in potential complications and cost of the procedure. However, autologous bone graft and bone lengthening with external fixation still remain the standard for treating trauma-related bone defects, where immediate 3D printing is not possible due to a long scaffold designing and printing time.

The review only reported the osteogenic outcomes of unmodified Ti64 scaffold, which could act as a guide for comparison with other porous biomaterials in future studies. However, the impact of scaffold's surface modification for improving its biological activity and osteogenic capacity cannot be ignored. Surface modification techniques such as anodic oxidation and micro-arc oxidation increase the surface area of the bone in contact with the oxidized scaffold, leading to a quicker and firmer integration of the implant $[60,61]$. Hydroxyapatite (HA) coating has also been widely used as a coating agent which demonstrated improved osseointegration and bone ingrowth compared to an uncoated Ti64 scaffold [20, 41]. Furthermore, ionsubstituted HA coatings (strontium and silicon substituted HA coatings) also offer an increased bone growth compared to pure HA [49]. Other coatings that have also shown to improve bone ingrowth, wear resistance, and osteogenic capacity of the scaffold include polydopamine coating [40], magnesium-calcium silicate composite coating [56], osteostatin coating [58], Sr-incorporated zeolite coating [59], and titanium-copper/titanium-copper nitride multilayer [36]. Apart from surface modifying techniques and coatings, the Ti64 scaffold has also been tailored with growth factors such as bone morphogenetic protein-2 (BMP-2) and vascular endothelial growth factor (VEGF) for inducing osteogenesis and angiogenesis, respectively $[41,45,57]$. In summary, all the aforementioned factors should be considered when designing a Ti64 scaffold to allow for optimal performance.

Based on the findings of the review, future in vivo studies are warranted using large animal models. Although small animals are acceptable for proving general principles, repeated experiments with larger animal models are necessary for translating the results to humans. Furthermore, as the mechanism of bone regeneration is also dependent on the size of the bone defect, it constitutes a need for creating large sized defects that can only be set in large animal models. Future research should also be conducted with a long-term follow-up period for assessing the precise osteogenic capacity of a Ti64 scaffold. It 
is also recommended to follow the ISO guidelines for designing experiments and standardized parameters such as $\mathrm{BA} / \mathrm{TA}, \mathrm{BV} / \mathrm{TV}$, and bone-implant-contact should be assessed for quantifying bone ingrowth. These proposed recommendations can greatly reduce data heterogeneity and improve study comparability.

At present, Ti64 scaffold is still a black box for researchers and further exploration is required to unravel its full potential. For instance, a more advanced functional Ti64 scaffold hierarchical design could be fabricated by combining different techniques such as topology optimization, CAD, and minimal surface formulation [9]; Furthermore, surface nano-topography modification could endow Ti64 scaffolds with additional biological functions such as antibacterial properties and enhanced osteogenic differentiation [79]. Another avenue of investigation could be the improvement in the AM processes to address the issues of unmelted powder particles and pores in the scaffold trabecula, which would negatively impact the scaffold's mechanical properties.

The review had certain limitations. Firstly, no standardization existed related to the pore size, porosity, strut size and pore shape among different studies. As all the parameters are correlated, thereby it was difficult to determine the best combination for designing the Ti64 scaffold. Secondly, most studies used rabbit's femoral epiphysis as the implantation site and a $5 \mathrm{~mm}$ diameter scaffold, which is too large for a rabbit model and also it does not represent the functional Ti64 scaffold used in humans. However, it should be noted that a scaffold with a smaller diameter is difficult to manufacture by the available 3D printing techniques. Finally, the longest follow-up time-point reported by majority of the studies was 12 weeks; hence, it was difficult to predict whether and when the new bone would outgrow the Ti64 scaffold.

\section{Conclusion}

Ti64 scaffold could act as a promising medium for providing mechanical support and a stable environment for new bone formation in long bone defects. Furthermore, rhombic dodecahedron- or diamond-shaped pores with a pore size of $500-700 \mu \mathrm{m}$ and $60-70 \%$ porosity could be considered as the most optimal parameters for manufacturing the scaffold. Further studies are required using large animal models and standardized protocols for extrapolating the results of animal studies to humans for potential clinical applications.

\footnotetext{
Abbreviations

Ti6Al4V: Titanium-6 aluminium-4 vanadium; Ti64 scaffold: 3D-printed porous Ti6Al4V scaffold; CAD: Computer-aided design; AM: Additive manufacturing; SLM: Selective laser melting; EBM: Electron beam melting; SLS: Selective laser sintering; PRISMA: Preferred Reporting Items for Systematic Reviews and MetaAnalyses; SEM: Scanning electron microscopy; BA/TA: Bone area fraction; BV/
}

TV: Bone volume fraction; HA: Hydroxyapatite; BMP-2: Bone morphogenetic protein-2; VEGF: Vascular endothelial growth factor.

\section{Supplementary Information}

The online version contains supplementary material available at https://doi. org/10.1186/s13018-022-02960-6.

Additional file 1. The detailed search strings in this study.

Additional file 2. The PRISMA checklist.

\section{Acknowledgements}

We acknowledge contributions of all members of the OMFS-IMPATH Research Group to this article.

\section{Authors' contributions}

YG and YS conducted the research and screening, extracted all of the data, and wrote the manuscript. SS assisted with the manuscript review and language editing. $A B$ and $C P$ assisted in reviewing and revising the manuscript. RJ oversaw the study's organization, screening, and manuscript review. All authors read and approved the final manuscript.

\section{Funding}

Open access funding provided by Karolinska Institute. This research did not receive any specific grant from funding agencies in the public, commercial, or not-for-profit sectors.

\section{Availability of data and materials}

The datasets used and/or analyzed during the current study are available from the corresponding author on reasonable request.

\section{Declarations}

Ethics approval and consent to participate Not applicable.

\section{Consent for publication}

Not applicable.

\section{Competing interests}

The authors declare that they have no competing interests.

\section{Author details}

${ }^{1}$ OMFS-IMPATH Research Group, Department of Imaging and Pathology, KU Leuven, Leuven, Belgium. ${ }^{2}$ Department of Oral and Maxillofacial Surgery, University Hospitals Leuven, Leuven, Belgium. ${ }^{3}$ Department of Materials Engineering, Biomaterials and Tissue Engineering Research Group, KU Leuven, 3000 Leuven, Belgium. ${ }^{4}$ Department of Dental Medicine, Karolinska Institutet, Stockholm, Sweden.

Received: 18 October 2021 Accepted: 21 January 2022

Published online: 02 February 2022

\section{References}

1. Warnke PH, Douglas T, Wollny P, Sherry E, Steiner M, Galonska S, et al. Rapid prototyping: porous titanium alloy scaffolds produced by selective laser melting for bone tissue engineering. Tissue Eng Part C Methods. 2008;15:115-24.

2. Tamimi F, Torres J, Al-Abedalla K, Lopez-Cabarcos E, Alkhraisat MH, Bassett DC, et al. Osseointegration of dental implants in 3D-printed synthetic onlay grafts customized according to bone metabolic activity in recipient site. Biomaterials. 2014;35:5436-45.

3. Li G, Wang L, Pan W, Yang F, Jiang W, Wu X, et al. In vitro and in vivo study of additive manufactured porous Ti6Al4V scaffolds for repairing bone defects. Sci Rep. 2016;6:34072 
4. Bandyopadhyay A, Mitra I, Shivaram A, Dasgupta N, Bose S. Direct comparison of additively manufactured porous titanium and tantalum implants towards in vivo osseointegration. Addit Manuf. 2019;28:259-66.

5. Zimmer. https://www.zimmerbiomet.com/en/products-and-solutions/ specialties/spine/trelloss-tc-porous-ti-interbody-system.html. Accessed 31 Dec 2021.

6. Zimmer. https://www.zimmerbiomet.com/en/products-and-solutions/ specialties/hip/osseoti-porous-metal-technology.html. Accessed 31 Dec 2021.

7. Nuvasive I. https://www.nuvasive.com/Surgical-Solutions/AdvancedMaterials-Science/Modulus-Titanium-Technology/. Accessed 31 Dec 2021

8. Spineart. https://www.spineart.com/products/juliet-ti-tl/. Accessed 31 Dec 2021

9. Rodriguez-Contreras A, Punset M, Calero JA, Gil FJ, Ruperez E, Manero JM. Powder metallurgy with space holder for porous titanium implants: a review. J Mater Sci Technol. 2021;76:129-49.

10. Elias C, Lima J, Valiev R, Meyers M. Biomedical applications of titanium and its alloys. JOM. 2008;60:46-9.

11. Zhang X, Zheng G, Wang J, Zhang Y, Zhang G, Li Z, et al. Porous Ti6Al4V scaffold directly fabricated by sintering: preparation and in vivo experiment. J Nanomater. 2013;2013:1-7.

12. Davis N, Teisen J, Schuh C, Dunand D. Solid-state foaming of titanium by superplastic expansion of argon-filled pores. J Mater Res. 2001;16:1508-19.

13. Li J, Li S, Van Blitterswijk C, De Groot K. A novel porous Ti6Al4V: characterization and cell attachment. J Biomed Mater Res A. 2005;73:223-33.

14. Sing SL, An J, Yeong WY, Wiria FE. Laser and electron-beam powder-bed additive manufacturing of metallic implants: a review on processes, materials and designs. J Orthop Res. 2016;34:369-85.

15. Karageorgiou V, Kaplan D. Porosity of 3D biomaterial scaffolds and osteogenesis. Biomaterials. 2005;26:5474-91.

16. Hara D, Nakashima Y, Sato T, Hirata M, Kanazawa M, Kohno Y, et al. Bone bonding strength of diamond-structured porous titanium-alloy implants manufactured using the electron beam-melting technique. Mater Sci Eng C. 2016;59:1047-52

17. Van der Stok J, Van der Jagt OP, Amin Yavari S, De Haas MF, Waarsing JH, Jahr $\mathrm{H}$, et al. Selective laser melting-produced porous titanium scaffolds regenerate bone in critical size cortical bone defects. J Orthop Res. 2013:31:792-9.

18. Fojt J. Ti-6Al-4V alloy surface modification for medical applications. Appl Surf Sci. 2012:262:163-7.

19. Tanzer M, Chuang PJ, Ngo CG, Song L, TenHuisen KS. Characterization of bone ingrowth and interface mechanics of a new porous 3D printed biomaterial: an animal study. Bone Jt J. 2019;6(Suppl B):62-7.

20. Huang H, Lan PH, Zhang YQ, Li XK, Zhang X, Yuan CF, et al. Surface characterization and in vivo performance of plasma-sprayed hydroxyapatitecoated porous Ti6Al4V implants generated by electron beam melting. Surf Coat. 2015:283:80-8.

21. Guo Y, Wu J, Xie K, Tan J, Yang Y, Zhao S, et al. Study of bone regeneration and osteointegration effect of a novel selective laser-melted titaniumtantalum-niobium-zirconium alloy scaffold. ACS Biomater Sci Eng. 2019;5:6463-73.

22. Guo Y, Xie K, Jiang W, Wang L, Li G, Zhao S, et al. In vitro and in vivo study of 3D-printed porous tantalum scaffolds for repairing bone defects. ACS Biomater Sci Eng. 2019;5:1123-33.

23. Wu S, Liu X, Yeung KW, Liu C, Yang X. Biomimetic porous scaffolds for bone tissue engineering. Mater Sci Eng. 2014;80:1-36.

24. Hooijmans CR, Rovers MM, De Vries RB, Leenaars M, Ritskes-Hoitinga M, Langendam MW. SYRCLE's risk of bias tool for animal studies. BMC Med Res Methodol. 2014;14:43

25. Davies KS. Formulating the evidence based practice question: a review of the frameworks. Evid Based Libr Inf Pract. 2011;6:75-80.

26. Ragone V, Canciani E, Arosio M, Olimpo M, Piras LA, von Degerfeld MM, et al. In vivo osseointegration of a randomized trabecular titanium structure obtained by an additive manufacturing technique. J Mater Sci Mater Med. 2020;31:17.

27. Wang H, Su K, Su L, Liang P, Ji P, Wang C. The effect of 3D-printed Ti6Al4V scaffolds with various macropore structures on osteointegration and osteogenesis: a biomechanical evaluation. J Mech Behav Biomed Mater 2018:88:488-96.
28. Yu T, Gao H, Liu T, Huang YD, Wang C. Effects of immediately static loading on osteointegration and osteogenesis around 3D-printed porous implant: a histological and biomechanical study. Mater Sci Eng C. 2020;108:110406.

29. Li L, Shi J, Zhang K, Yang L, Yu F, Zhu L, et al. Early osteointegration evaluation of porous Ti6Al4V scaffolds designed based on triply periodic minimal surface models. J Orthop Transl. 2019:19:94-105.

30. Arabnejad S, Johnston RB, Pura JA, Singh B, Tanzer M, Pasini D. Highstrength porous biomaterials for bone replacement: a strategy to assess the interplay between cell morphology, mechanical properties, bone ingrowth and manufacturing constraints. Acta Biomater. 2016;30:345-56.

31. Chen C, Hao Y, Bai X, Ni JJ, Chung SM, Liu F, et al. 3D printed porous Ti6Al4V cage: effects of additive angle on surface properties and biocompatibility; bone ingrowth in Beagle tibia model. Mater Des. 2019;175:107824

32. Chen Z, Yan X, Yin S, Liu L, Liu X, Zhao G, et al. Influence of the pore size and porosity of selective laser melted Ti6Al4V ELI porous scaffold on cell proliferation, osteogenesis and bone ingrowth. Mater Sci Eng C. 2020;106:110289

33. Crovace AM, Lacitignola L, Forleo DM, Staffieri F, Francioso E, Di Meo A, et al. 3D biomimetic porous titanium (Ti6Al4V ELI) scaffolds for large bone critical defect reconstruction: an experimental study in sheep. Animals. 2020;10:1389.

34. Fan B, Guo Z, Li X, Li S, Gao P, Xiao X, et al. Electroactive barium titanate coated titanium scaffold improves osteogenesis and osseointegration with low-intensity pulsed ultrasound for large segmental bone defects. Bioact Mater. 2020;5:1087-101.

35. Gilev MV, Bazarny W, Volokitina EA, Polushina LG, Maksimova AY, Kazakova YE. Laboratory monitoring of bone tissue remodeling after augmentation of impression intraarticular fracture with different types of bone graft. Bull Exp Biol Med. 2019;167:681-4.

36. Guo Y, Ren L, Xie K, Wang L, Yu B, Jiang W, et al. Functionalized TiCu/Ti$\mathrm{Cu}-\mathrm{N}$-coated 3D-printed porous Ti6Al4V scaffold promotes bone regeneration through BMSC recruitment. Adv Mater Interfaces. 2020;7:1901632.

37. Han TX, Chang B, Ding X, Yue GN, Song W, Tang HP, et al. Improved bone formation and ingrowth for additively manufactured porous Ti6Al4V bone implants with strontium laden nanotube array coating. RSC Adv. 2016:6:13686-97.

38. Kelly CN, Lin AS, Leguineche KE, Shekhar S, Walsh WR, Guldberg RE, et al. Functional repair of critically sized femoral defects treated with bioinspired titanium gyroid-sheet scaffolds. J Mech Behav Biomed Mater. 2021:116:104380.

39. Koolen M, Amin Yavari S, Lietaert K, Wauthle R, Zadpoor AA, Weinans H. Bone regeneration in critical-sized bone defects treated with additively manufactured porous metallic biomaterials: the effects of inelastic mechanical properties. Materials. 2020;13:1992.

40. Li L, Li Y, Yang L, Yu F, Zhang K, Jin J, et al. Polydopamine coating promotes early osteogenesis in 3D printing porous Ti6Al4V scaffolds. Ann Transl Med. 2019;7:240.

41. Li Y, Yang W, Li X, Zhang X, Wang C, Meng X, et al. Improving osteointegration and osteogenesis of three-dimensional porous Ti6Al4V scaffolds by polydopamine-assisted biomimetic hydroxyapatite coating. ACS Appl Mater Interfaces. 2015;7:5715-24.

42. Liu H, Li W, Liu C, Tan J, Wang H, Hai B, et al. Incorporating simvastatin/ poloxamer 407 hydrogel into 3D-printed porous Ti6Al4V scaffolds for the promotion of angiogenesis, osseointegration and bone ingrowth. Biofabrication. 2016;8:045012.

43. Liu LZ, Duan JH, Shi Q, Chen Q, Yao QQ, Li ZY. Mechanical effect on the evolution of bone formation during bone ingrowth into a 3D-printed Ti-alloy scaffold. Mater Lett. 2020;273:127971.

44. Luan HQ, Wang LT, Ren WY, Chu ZW, Huang YF, Lu CL, et al. The effect of pore size and porosity of Ti6Al4V scaffolds on MC3T3-E1 cells and tissue in rabbits. Sci China Technol Sci. 2019;62:1160-8.

45. Lv J, Xiu P, Tan J, Jia Z, Cai H, Liu Z. Enhanced angiogenesis and osteogenesis in critical bone defects by the controlled release of BMP-2 and VEGF: implantation of electron beam melting-fabricated porous Ti6Al4V scaffolds incorporating growth factor-doped fibrin glue. Biomed Mater. 2015;10:035013. 
46. Lyu LW, Jing Y, Wang JK, Zhang CQ. Enhanced osseointegration of porous titanium scaffold implanted with preload: an experiment study in rabbits. Int J Morphol. 2020;38:909-13.

47. Ma L, Wang X, Zhao N, Zhu Y, Qiu Z, Li Q, et al. Integrating 3D printing and biomimetic mineralization for personalized enhanced osteogenesis, angiogenesis, and osteointegration. ACS Appl Mater Interfaces. 2018;10:42146-54.

48. Ma L, Wang X, Zhou Y, Ji X, Cheng S, Bian D, et al. Biomimetic Ti-6Al-4V alloy/gelatin methacrylate hybrid scaffold with enhanced osteogenic and angiogenic capabilities for large bone defect restoration. Bioact Mater. 2021;6:3437-48.

49. Mumith A, Cheong VS, Fromme P, Coathup MJ, Blunn GW. The effect of strontium and silicon substituted hydroxyapatite electrochemical coatings on bone ingrowth and osseointegration of selective laser sintered porous metal implants. PLoS ONE. 2020;15:e0227232

50. Palmquist A, Shah FA, Emanuelsson L, Omar O, Suska F. A technique for evaluating bone ingrowth into 3D printed, porous Ti6AI4V implants accurately using X-ray micro-computed tomography and histomorphometry. Micron. 2017:94:1-8.

51. Palmquist A, Snis A, Emanuelsson L, Browne M, Thomsen P. Long-term biocompatibility and osseointegration of electron beam melted, freeform-fabricated solid and porous titanium alloy: experimental studies in sheep. J Biomater Appl. 2013;27:1003-16.

52. Ran Q, Yang W, Hu Y, Shen X, Yu Y, Xiang Y, et al. Osteogenesis of 3D printed porous Ti6Al4V implants with different pore sizes. J Mech Behav Biomed Mater. 2018;84:1-11.

53. Shah FA, Snis A, Matic A, Thomsen P, Palmquist A. 3D printed Ti6Al4V implant surface promotes bone maturation and retains a higher density of less aged osteocytes at the bone-implant interface. Acta Biomater. 2016;30:357-67.

54. Shah FA, Omar O, Suska F, Snis A, Matic A, Emanuelsson L, et al. Long-term osseointegration of $3 \mathrm{D}$ printed $\mathrm{CoCr}$ constructs with an interconnected open-pore architecture prepared by electron beam melting. Acta Biomater. 2016;36:296-309.

55. Song P, Hu C, Pei X, Sun J, Sun H, Wu L, et al. Dual modulation of crystallinity and macro-/microstructures of 3D printed porous titanium implants to enhance stability and osseointegration. J Mater Chem B. 2019;7:2865-77.

56. Tsai $\mathrm{CH}$, Hung $\mathrm{CH}$, Kuo $\mathrm{CN}$, Chen $\mathrm{CY}$, Peng $\mathrm{YN}$, Shie MY. Improved bioactivity of 3D printed porous titanium alloy scaffold with chitosan/magnesium-calcium silicate composite for orthopaedic applications. Materials. 2019;12:203.

57. van der Stok J, Koolen MK, de Maat MP, Yavari SA, Alblas J, Patka P, et al. Full regeneration of segmental bone defects using porous titanium implants loaded with BMP-2 containing fibrin gels. Eur Cell Mater. 2015:29:141-53.

58. van der Stok J, Lozano D, Chai YC, Amin Yavari S, Bastidas Coral AP, Verhaar JA, et al. Osteostatin-coated porous titanium can improve early bone regeneration of cortical bone defects in rats. Tissue Eng Part A. 2015;21:1495-506.

59. Wang S, Li R, Li D, Zhang ZY, Liu G, Liang H, et al. Fabrication of bioactive 3D printed porous titanium implants with Sr ion-incorporated zeolite coatings for bone ingrowth. J Mater Chem B. 2018:6:3254-61.

60. Xiu P, Jia ZJ, LV J, Yin C, Cai H, Song CL, et al. Hierarchical micropore/ nanorod apatite hybrids in situ grown from 3-D printed macroporous Ti6Al4V implants with improved bioactivity and osseointegration. J Mater Sci Technol. 2017;33:179-86.

61. Xiu P, Jia Z, LV J, Yin C, Cheng Y, Zhang K, et al. Tailored surface treatment of 3D printed porous Ti6Al4V by microarc oxidation for enhanced osseointegration via optimized bone in-growth patterns and interlocked bone/implant interface. ACS Appl Mater Interfaces. 2016;8:17964-75.

62. Amin Yavari S, van der Stok J, Chai YC, Wauthle R, Tahmasebi Birgani Z, Habibovic $P$, et al. Bone regeneration performance of surface-treated porous titanium. Biomaterials. 2014;35:6172-81.

63. Zhang G, Zhao P, Lin L, Qin L, Huan Z, Leeflang S, et al. Surface-treated 3D printed Ti-6Al-4V scaffolds with enhanced bone regeneration performance: an in vivo study. Ann Transl Med. 2021;9:39.

64. Zhong W, Li J, Hu C, Quan Z, Jiang D, Huang G, et al. 3D-printed titanium implant-coated polydopamine for repairing femoral condyle defects in rabbits. J Orthop Surg Res. 2020;15:102.
65. von Rechenberg B. Animal models in bone repair. Drug Discov Today Dis Models. 2014;13:23-7.

66. Pearce A, Richards R, Milz S, Schneider E, Pearce S. Animal models for implant biomaterial research in bone: a review. Eur Cell Mater. 2007;13:1-10.

67. Reichert JC, Saifzadeh S, Wullschleger ME, Epari DR, Schütz MA, Duda GN, et al. The challenge of establishing preclinical models for segmental bone defect research. Biomaterials. 2009;30:2149-63.

68. Hakimi M, Jungbluth $P$, Sager M, Betsch M, Herten M, Becker J, et al. Combined use of platelet-rich plasma and autologous bone grafts in the treatment of long bone defects in mini-pigs. Injury. 2010;41:717-23.

69. Histing T, Garcia P, Holstein J, Klein M, Matthys R, Nuetzi R, et al. Small animal bone healing models: standards, tips, and pitfalls results of a consensus meeting. Bone. 2011;49:591-9.

70. Willie BM, Bloebaum RD, Bireley WR, Bachus KN, Hofmann AA. Determining relevance of a weight-bearing ovine model for bone ingrowth assessment. J Biomed Mater Res A. 2004;69:567-76.

71. Wancket L. Animal models for evaluation of bone implants and devices: comparative bone structure and common model uses. Vet Pathol. 2015;52:842-50.

72. Mangione F, Salmon B, EzEldeen M, Jacobs R, Chaussain C, Vital S. Characteristics of large animal models for current cell-based oral tissue regeneration. Tissue Eng Part B Rev. 2021. https://doi.org/10.1089/ten.teb. 2020.0384

73. McGovern JA, Griffin M, Hutmacher DW. Animal models for bone tissue engineering and modelling disease. Dis Model Mech. 2018;11:033084.

74. Li Y, Chen S-K, Li L, Qin L, Wang X-L, Lai Y-X. Bone defect animal models for testing efficacy of bone substitute biomaterials. J Orthop Translat. 2015;3:95-104.

75. Surmeneva MA, Surmenev RA, Chudinova EA, Koptioug A, Tkachev MS, Gorodzha SN, et al. Fabrication of multiple-layered gradient cellular metal scaffold via electron beam melting for segmental bone reconstruction. Mater Des. 2017;133:195-204.

76. DuY, Guo JL, Wang J, Mikos AG, Zhang S. Hierarchically designed bone scaffolds: from internal cues to external stimuli. Biomaterials. 2019;218:119334.

77. Zhang $X-Y$, Fang $G$, Xing L-L, Liu W, Zhou J. Effect of porosity variation strategy on the performance of functionally graded Ti-6Al-4V scaffolds for bone tissue engineering. Mater Des. 2018;157:523-38.

78. Huang G, Pan S-T, Qiu J-X. The osteogenic effects of porous tantalum and titanium alloy scaffolds with different unit cell structure. Colloids Surf B Biointerfaces. 2021;210:112229.

79. Hasan J, Jain S, Chatterjee K. Nanoscale topography on black titanium imparts multi-biofunctional properties for orthopedic applications. Sci Rep. 2017;7:1-13.

\section{Publisher's Note}

Springer Nature remains neutral with regard to jurisdictional claims in published maps and institutional affiliations.

Ready to submit your research? Choose BMC and benefit from:

- fast, convenient online submission

- thorough peer review by experienced researchers in your field

- rapid publication on acceptance

- support for research data, including large and complex data types

- gold Open Access which fosters wider collaboration and increased citations

- maximum visibility for your research: over $100 \mathrm{M}$ website views per year

At BMC, research is always in progress.

Learn more biomedcentral.com/submissions 